\title{
Monitoring of Extracellular TCA Cycle Intermediates in Mammalian Cell Culture
}

\author{
Joachim B. Ritter ${ }^{1}$, Yvonne Genzel ${ }^{1}$, Udo Reichl ${ }^{1,2}$ \\ ${ }^{1}$ Max Planck Institute for Dynamics of Complex Technical Systems, Magdeburg, Germany \\ ${ }^{2}$ Otto-von-Guericke-University Magdeburg, Chair of Bioprocess Engineering, Magdeburg, Germany
}

In recent years, monitoring of metabolites such as glucose, lactate, ammonia, glutamine, glutamate, and other amino acids has been introduced for many cell cultivations. Assaying intracellular metabolites might reveal further insights into metabolism. However, some of the mainly intracellular or even mitochondrial appearing metabolites can also be found in the medium supernatant at micromolar concentrations. Since no active transport mechanisms for excretion of these intermediates are known for most mammalian production cell lines, they might leak out of the cells and thus could be correlated to intracellular concentrations.

In this work, we are investigating extracellular concentrations of five organic acids (succinic, malic, fumaric, citric, isocitric acid) during growth looking at different media and cell lines. Moreover, clear influences on concentrations were also expected to occur after viral infection of MDCK cells.

The analysis is performed using an anion-exchange chromatography system (DX-320, Dionex, Idstein, Germany) with a conductivity detector. Due to high protein content of the medium, sample preparation posed some problems. Several strategies have been investigated for an optimal recovery of the abovementioned organic acids.

Experiments showed that certain amounts of these organic acids already occurred in fresh medium, coming from both serum and peptone being supplements for the culture medium. Nevertheless, first results indicate significant changes of concentrations during the course of cultivation, probably not correlated to dead cells. In batch cultivation, malic, fumaric and citric acid show an almost linear increase after the lag phase, whereas isocitric and succinic acid seem to reach a constant level during stationary phase. 